\title{
Cardiac rupture in post-myocardial infarction syndrome
}

\author{
W. S. HISLOP* \\ M.B., M.R.C.P. \\ Department of Cardiology, Western Infirmary, Glasgow
}

\begin{abstract}
Summary
A fatal case of post-myocardial infarction syndrome (PMIS) followed by a rupture of an inferior left ventricular aneurysm is described. Attention is drawn to the growing number of reports of aneurysm in PMIS, and the clinico-pathological significance of this association is discussed.

\section{Introduction}

The post-myocardial infarction syndrome, or Dressler's syndrome (after the physician who described it in 1959), consists of pericardial and pleuritic pain, fever, raised ESR, leucocytosis, pericardial rub, pericardial and pleural effusions and pneumonitis, usually from one week to several months following a myocardial infarction; the whole clinical picture having a tendency to relapse, but an excellent response to steroids.
\end{abstract}

\section{Case history}

A 68-year-old retired civil engineer consulted his general practitioner followjng one week's history of praecordial pain and dyspnoea related to moderate exertion. An out-patient ECG was reported normal, a diagnosis of angina pectoris made, and he was started on glyceryl trinitrate and propranolol. His symptoms got worse, however, and he consulted his practitioner again one month later with continual crushing retrosternal chest pain radiating to his left arm. His practitioner noticed he was pale, sweating, anxious and obviously unwell. Blood pressure was $165 / 85 \mathrm{mmHg}$. He was admitted to the Coronary Care Unit where the above history was verified. The only past history was of haemorrhoids.

On admission his blood pressure had fallen to $110 / 70 \mathrm{mmHg}$, and severe chest pain required diamorphine. An ECG confirmed an inferior myocardial infarction with lateral extension (Fig. 1), this being corroborated by a rise in serial cardiac enzymes. On the first evening his course was complicated by intermittent junctional bradycardia, transient left bundle branch block and idioventricular rhythm. Thereafter his course was uncomplicated except for transient left ventricular failure which

* Present address: Department of Medicine (University, of Dundee), Ninewells Hospital, Dundee DD2 1 UB. responded to a thiazide diuretic and fourteen days after admission he was discharged on thiazide, potassium and propranolol.

Eight days later he was re-admitted with further chest pain which was clearly different from that of his infarction, being sharper, radiating to both shoulders, exacerbated by deep breathing and associated with marked dyspnoea. A pulmonary embolism was suspected. Examination showed a blood pressure of $125 / 75 \mathrm{mmHg}$; jugular venous pressure (JVP) of $4 \mathrm{~cm}$, displacement of the apex beat $2 \mathrm{~cm}$ to the left, but there were no signs in the chest nor evidence of a deep vein thrombosis (DVT). ECG was unchanged.

Over the next few days persistent pyrexia $\left(38.4^{\circ} \mathrm{C}\right)$, signs of bilateral pleural effusions, and pericardial rub developed. ESR was $124 \mathrm{~mm} / \mathrm{hr}$, and white cell count consistently between 15.6 and $20.0 \times 10^{9} / 1$. A diagnosis of post-myocardial infarction syndrome was made. Prednisolone, $10 \mathrm{mg}$ t.i.d., was commenced five days after re-admission, and in five days his response was so good that the dosage was reduced, and his discharge was considered. At this time some palpable dyskinesia was noticed over the praecordium, and a ventricular aneurysm was considered a possible cause; the pericardial rub persisted, but there was clinical and radiological regression of the pleural effusions (Fig. 2).

Early on the day of his expected discharge his condition deteriorated. He was shocked (BP 75/0 $\mathrm{mmHg}$ ), pale, sweaty and he complained of severe anterior chest pain. JVP was grossly elevated, head and neck engorged and cyanosed. The pericardial rub had gone, S3 and S4 were now present with sinus tachycardia, and basal crepitations were marked. Following resuscitation he stabilized for some hours; CVP was found to be $22 \mathrm{~cm}$, pulsus paradoxus of 20 $\mathrm{mmHg}$ being also noted. There was no electrical alternans on ECG, but cardiac tamponade was considered to be present. Before paracentesis could be performed the patient died in asystolic cardiac arrest.

\section{Post-mortem findings}

The heart showed moderate left-ventricular hypertrophy, with an aneurysm on the postero-inferior 


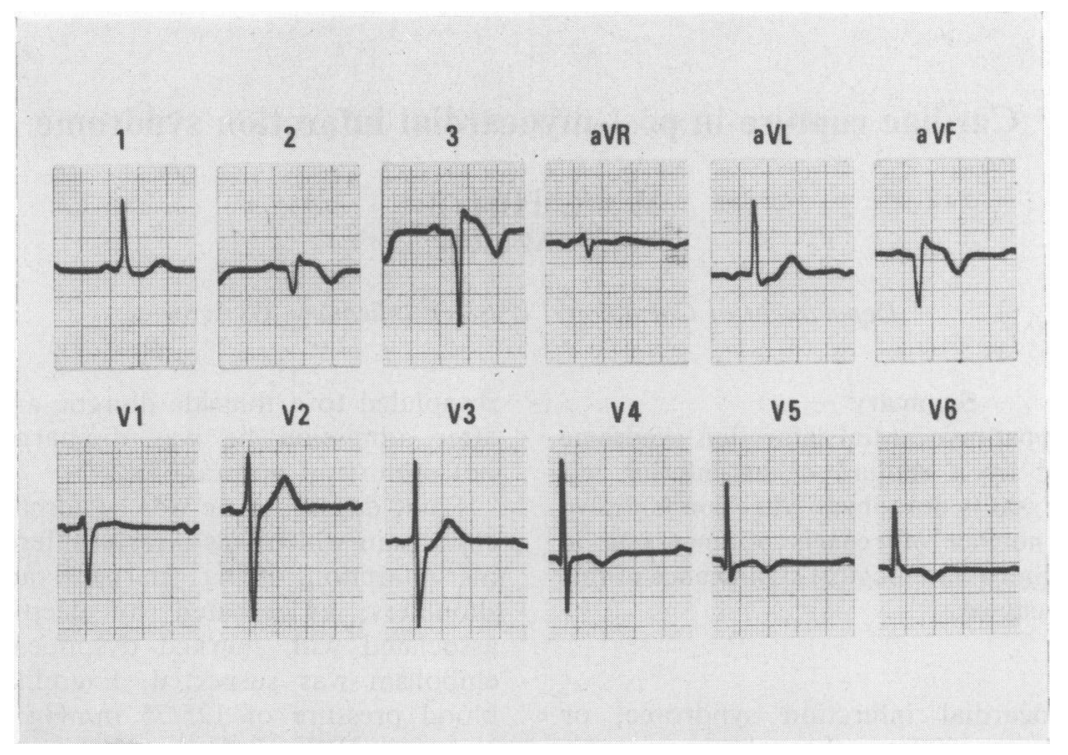

Fig. 1. Electrocardiogram confirming inferior myocardial infarction with lateral extension.

wall in an area of infarction of about six weeks' duration. This was ruptured and $400 \mathrm{ml}$ of blood were present in the pericardial sac with leakage into the mediastinum. There were widespread fibrinous pericardial adhesions. There was an occlusion of the right coronary artery with degenerate thrombus at its origin. Bilateral pleural effusions of about $500 \mathrm{ml}$ each were found. An incidental finding was the presence of two large pulmonary emboli, and dissection of the calf veins revealed a deep venous thrombosis. Histology of muscle adjacent to the infarcted area showed combined infarction and pericarditis (Fig. 3).

\section{Discussion}

The phenomenon of PMIS has caused controversy since Dressler's article in 1959. He described an incidence of $3-4 \%$ in all myocardial infarctions, but different authors (Broch and Ofstad, 1960; Davidson, Oliver and Robertson, 1961; Thadani et al., 1971; Niarchos and McKendrick, 1973) have reported an incidence of between nil and 3\%. The criteria for diagnosis, as mentioned earlier, seem well established, but Dressler stressed the distinction between PMIS and the pericarditis of acute myocardial infarction (caused by extension of the infarct to involve the epicardium); in particular, the later onset of PMIS (second to eleventh week of the illness) and the longer-lasting pericardial rub (three days to three weeks).

Dressler found the main complication of PMIS was haemorrhagic cardiac tamponade in patient receiving anticoagulant therapy, which caused ofre death of one member of his series. Most authors sided have felt that anticoagulants should be withdrawn i all forms of pericarditis following myocardial infar tion, although many workers dispute whether this necessary (Liem et al., 1975).

One complication or associated feature of PMI\$ not apparent to Dressler in 1959 appears to b8 ventricular aneurysm. In 1968, Ravnskov described two fatal cases of PMIS, with aneurysms detected only at post-mortem. In his review of literature however, he noted six further cases of aneurysm in nine autopsied fatal cases of PMIS, and severat reports of aneurysm in non-fatal PMIS cases. A case. of aneurysm developing after documented PMIS has been recorded (Berman and Grismer, 1970). By 197. thirteen deaths had been reported in PMIS, ten having ventricular aneurysms (Curry, 1972); this author stressed that this was a much higher propos. tion than would be expected by chance $(0.6 \%)$, an that clinicians should be aware of the association. $ᄋ$

Although aneurysm was suspected in the patienty reported here, this is not always the case; and hate the author been aware of the association alluded to above, the onset of PMIS might have prompted further investigation and possibly surgery to repa年 the aneurysm.

The aetiology of PMIS is thought to be autov immune in that auto-antibodies are directed agains $\bar{\Phi}$ certain components of damaged cardiac tissue, an $\$$ 

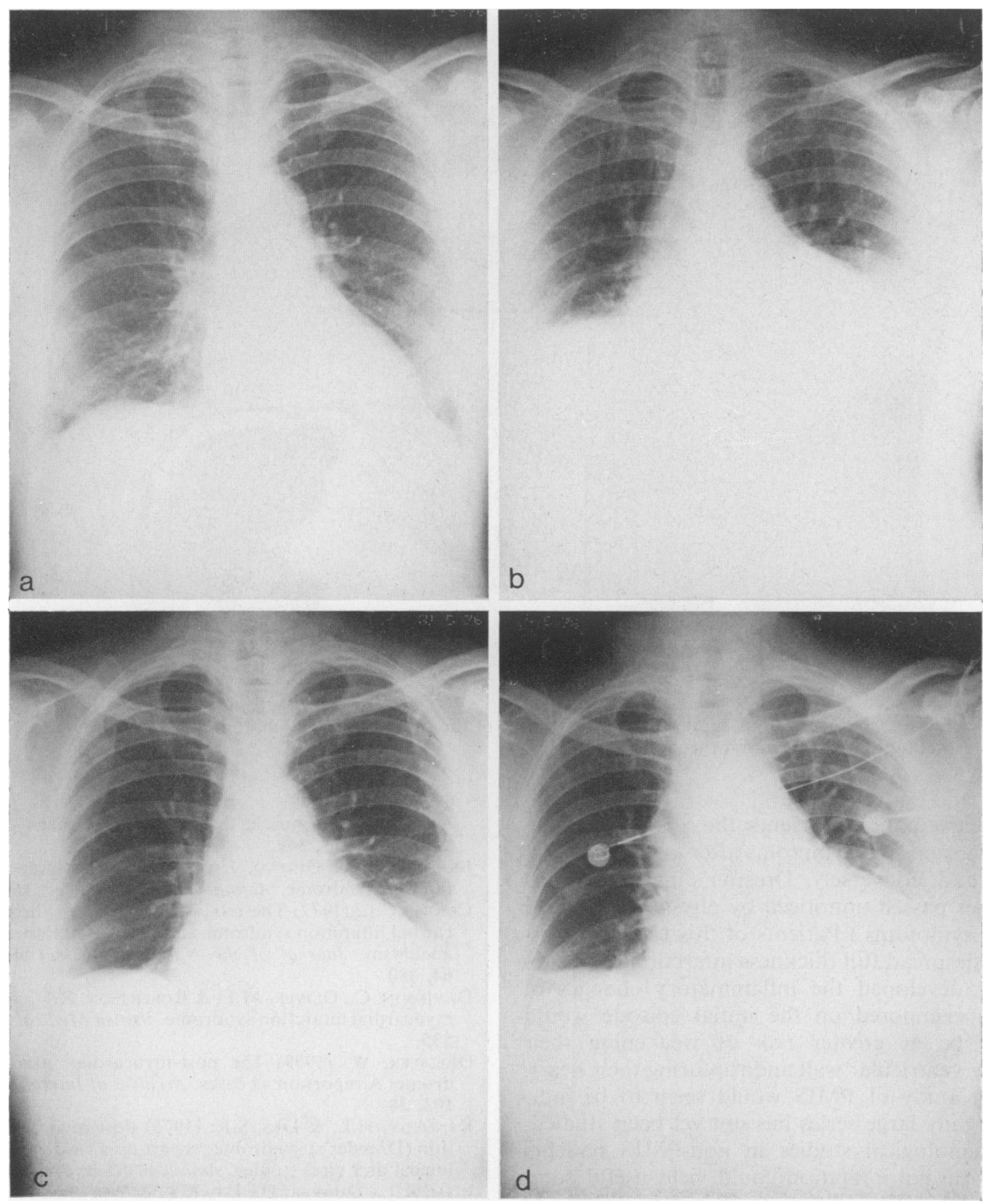

FIG. 2. (a) chest $\mathrm{X}$-ray at time of infarction, (b) chest $\mathrm{X}$-ray when presenting with post-myocardial infarction syndrome (PMIS), (c) after four days of prednisolone, $10 \mathrm{mg}$ t.i.d., (d) chest X-ray on day of cardiac rupture - note broadened mediastinum.

recent work has confirmed the presence of anti-heart antibodies of different kinds, and also increased numbers of lymphocytes containing surface IgG immunoglobulin in the acute stage of PMIS (Kennedy and Das, 1976).

What, then, is a possible basis for the association under discussion? It is known that not all post- myocardial infarction ventricular aneurysms occur in conjunction with PMIS, it is also known that not all full thickness infarctions develop aneurysm. The aetiology postulated above could mean that the autoimmune process outlined might take place subclinically in many individuals, and that PMIS is but a manifestation of this process in immunologically 


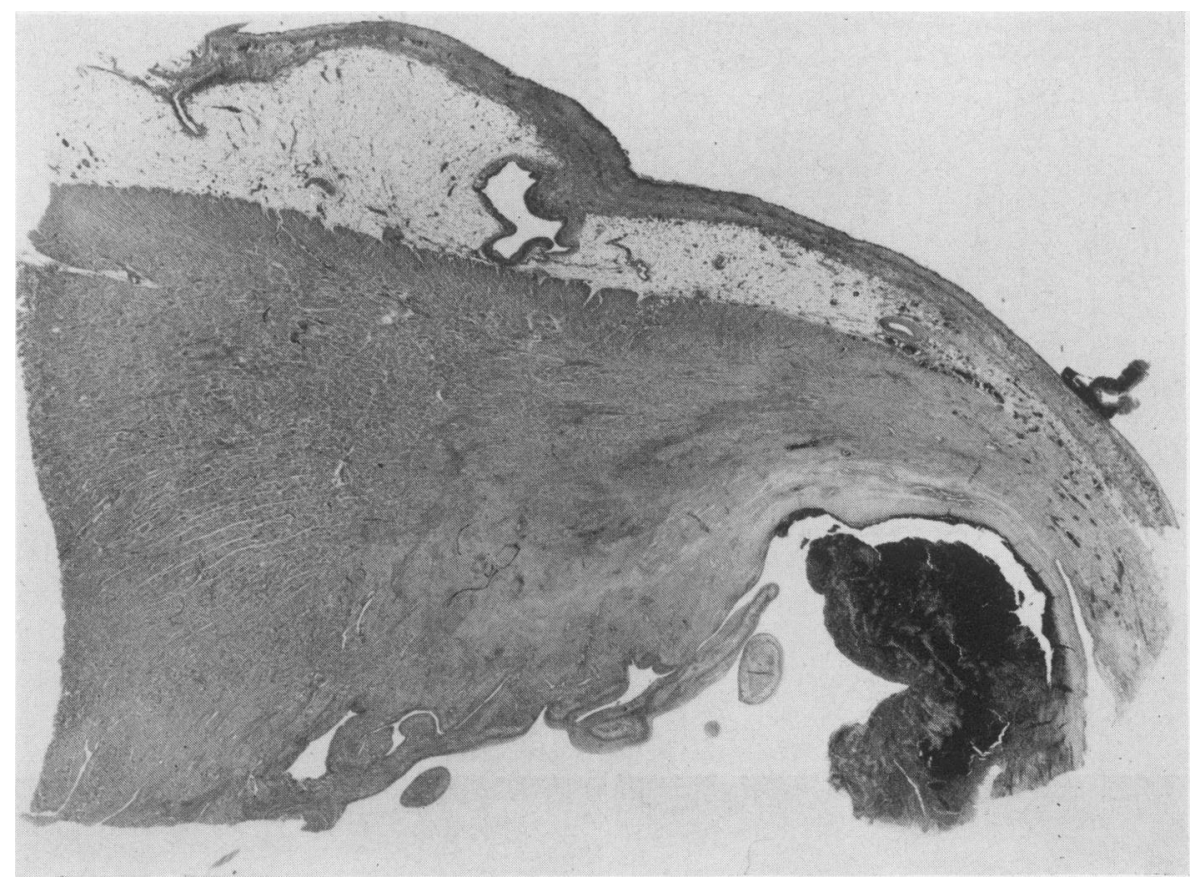

Fig. 3. Cross-section of heart muscle near the site of the rupture showing thinning of the ventricular wall and adherent mural thrombus.

hyper-reactive patients. (Hence the reports of PMIS with florid signs and symptoms after a very small infarction, and, conversely, Dressler's impression that many cases passed unnoticed by physicians because of trivial symptoms.) Patients of this type who sustained widespread full thickness infarctions and subsequently developed the inflammatory changes of PMIS superimposed on the initial episode would therefore be at greater risk of weakening their remaining ventricular wall and rupturing their heart.

Further study of PMIS would seem to be indicated, a really large series has not yet been studied, and immunological studies in non-PMIS patients after myocardial infarction could yield useful comparative data. For clinicians, however, it would appear that PMIS can be more than just a trivial complication of a common disorder, it could indicate an undetected ventricular aneurysm, and where an aneurysm is suspected, early surgical intervention may be advisable.

\section{References}

Berman, D.A. \& Grismer, J.T. (1970) Post-myocardial infarction syndrome followed by left ventricular aneurysm with papillary muscle dysfunction. American Journal $\stackrel{\sum}{\Phi} f$ Cardiology, 25, 349.

BRoch, O.J. \& OFSTAD, J. (1960) The post-myocardial 耑farction syndrome. Acta medica scandinavica, 166, 281. Ф

CurRY, C.L. (1972) The relationship between the post-myocardial infarction syndrome and post-infarction ventricuê.r aneurysm. Journal of the National Medical Association, 64,480 .

Davidson, C., Oliver, M.F. \& Robertson, R.F. (1961) Pof myocardial infarction syndrome. British Medical Journal, 2 , 535.

DRESSLER, W. (1959) The post-myocardial infarction sydrome. A report on 44 cases. Archives of Internal Medici度, 103, 28.

KENNEDY, H.L. \& DAS, S.K. (1976) Post-myocardial infarition (Dressler's) syndrome: report on a case with immunilogical and viral studies. American Heart Journal, 91, 233.

Liem, K.L., Durrer, D., Lie, K.L. \& Wellens, H.J.J. (197) Pericarditis in acute myocardial infarction. Lancet, 1004.

Niarchos, A.P. \& McKendrick, C.S. (1973) Prognosis pericarditis after acute myocardial infarction. Brithogh Heart Journal, 35, 49.

RavNSKov, U. (1968) Aneurysm of the heart and the pospmyocardial infarction syndrome. Acta medica scandinaving, 183, 393.

Thadani, U., Chopra, M.P., Aber, C.P. \& Portal, R. (1971) Pericarditis after acute myocardial infarction. British Medical Journal, 2, 135. 